\title{
Los claroscuros de transferencias intergubernamentales y la eficiencia relativa: caso éstado de Jalisco, México
}

The chiaroscuro of intergovernmental transfer and the relative efficiency: Jalisco, México case

\author{
Filadelfo León Cázares* \\ Ana E. Ortiz Medina** \\ Daniel A. Cernas Ortiz****
}

\section{Resumen}

El objetivo de este estudio es estimar la eficiencia relativa de los municipios del estado mexicano de Jalisco y evaluar su relación con las transferencias intergubernamentales para los años 1990, 2000, y 2010. Se calcula la eficiencia relativa de los municipios a través de la metodología Data Envelopment Analysis (DEA). Los supuestos del análisis de medición de eficiencia se enfocan en la minimización de insumos con economías de escala variables. Los insumos considerados son servicios personales (SP), materiales y suministros (MYS), servicios generales $(\mathrm{SG})$. Los productos son la inversión pública (IP), ingresos tributarios y no tributarios. Por comparabilidad las variables monetarias se expresan a precios constantes per cápita. Encontramos que no hay diferencias estadísticas entre los municipios eficientes e ineficientes en cuanto a la asignación de las transferencias. Los resultados sugieren que no hay incentivos a los municipios ineficientes para cambiar su status. Adicionalmente, pareciera que existe potencialidad para incrementar la captación de los ingresos no tributarios en los municipios jaliscienses.

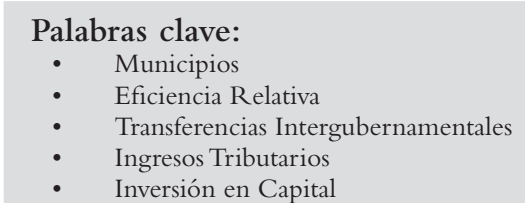

\section{Abstract}

The objective of this study is to estimate the relative efficiency of the municipalities of the Mexican State of Jalisco and evaluate its relationship with the intergovernmental grants in 1990, 2000, and 2010. The relative efficiency of the municipalities is estimated using Data Envelopment Analysis (DEA) methodology. The assumptions of efficiency measurement analysis focus on the minimization of inputs with variable returns to scale. The considered inputs are personnel services, material and other inputs, and general services. Outputs are capital investment and tax and fees collection. By comparability the monetary variables are expressed at constant prices per capita. We found no statistical difference between efficient and inefficient municipalities regarding to intergovernmental grants. The results suggest that there is no incentive for inefficient municipalities to change their status. Additionally, results suggest that municipalities of the State of Jalisco are having an increase on their incomes due to fees.

JEL: H21, H77, R11, R58

\section{Introducción}

Al considerarse el Federalismo Fiscal, dentro de este trabajo, como "el arreglo espacial del poder reconocido y garantizado por la Constitución Política y

*Departamento de Métodos Cuantitativos, Universidad de Guadalajara. <filadelfo@cucea.udg.mx> **Departamento de Estudios Políticos, Universidad de Guadalajara. <ana.ortiz@cush.udg.mx> ***Facultad de Contaduría y Administración, Universidad Autónoma del Estado de México. 
Economía Informa núm. 393 julio - agosto • 2015

expresado a través de las finanzas públicas y como función pública representativa del poder político" (Mandujano, 2011, p. 21). Dicho arreglo, de acuerdo a Mandujano, consiste en asignar responsabilidades ${ }^{1}$ a los diferentes niveles de gobierno que, por lo tanto, a los gobiernos locales les correspondería, de acuerdo al Artículo 115 Constitucional, imponer tributos, recaudar y administrar los recursos fiscales y además tener la facultad de proveer los bienes públicos que demanden los ciudadanos. Sin embargo, al ser insuficientes los recursos para financiar dichas responsabilidades, normalmente se generan desequilibrios verticales y horizontales dentro de las finanzas de los diferentes órdenes de gobierno (Ahmad y Craig, 1997). Por un lado, de acuerdo a estos autores, el desequilibrio vertical se da cuando los ingresos propios y gasto de los diferentes órdenes de gobierno son desiguales. Por otro lado, el desequilibrio horizontal ocurre cuando las capacidades fiscales de los órdenes de gobierno son diferentes.

En la literatura (e.g., Ahmad y Craig 1997; Velázquez 2003, y Cabrera 2010) se argumenta que las transferencias ${ }^{2}$ intergubernamentales, son un mecanismo importante para reducir estos desequilibrios con la finalidad de que los gobiernos, especialmente los locales, puedan cumplir con las demandas de sus gobernados. De hecho Trujillo (2008) argumenta que el objetivo de las transferencias es la búsqueda de la eficiencia y la equidad interjurisdicional, sin embargo puede darse el caso de que no se llegue a cumplir con dicho objetivo.

Cabrera y Lozano (2010) argumentan que la problemática para el caso mexicano es la existencia de varios fondos de transferencias que hacen compleja su administración, además de que no hay una suficiente claridad en los objetivos, ya que se combinan fondos condicionados e incondicionados. También, las transferencias no incorporan explícitamente, ni la medición de la necesidad del gasto ni de la capacidad fiscal de los gobiernos subnacionales. Por ende, esta situación puede inhibir la eficiencia de los gobiernos locales que podría verse reflejada, por ejemplo, en una dependencia hacia las trasferencias que, como consecuencia, generaría pereza fiscal en los municipios mexicanos sobre todo en los más pequeños y con una mayor marginación (Noguez, 2006).

\footnotetext{
${ }^{1}$ La transferencia o asignación de responsabilidades de gobierno central a los gobiernos subnacionales también llamado devolución o descentralización (Cabrera y Lozano, 2010). La asignación de responsabilidades ayuda a la eficiencia de los gobiernos a través de una mejor estimación de la demanda de los servicios públicos (Ahmad y Ruggiero, 1997).

${ }^{2}$ Algunos autores también las nombran como ingresos trasferidos (Cabrera y Lozano, 2010). Para fines de este trabajo se consideran las transferencias o recursos transferidos como la suma de las participaciones (no condicionadas) y aportaciones (condicionadas) que son reportadas en El Sistema Estatal y Municipal de Base de Datos (SIMBAD).
} 
Adicionalmente, en la literatura de las finanzas públicas se reconoce que las transferencias intergubernamentales propician el flypaper effect o efecto papel matamoscas. Esto es, los gobiernos subnacionales incrementan su gasto público en una proporción mayor cuando reciben transferencias que cuando usan sus recursos propios. Este argumento es probado empíricamente por Sour (2013) donde presenta evidencia del efecto papel matamoscas de las trasferencias no condicionadas con los gobiernos municipales durante el periodo 1990 a 2007.

Las consecuencias de los argumentos anteriores muestran la poca efectividad de las transferencias intergubernamentales en el cumplimiento de sus objetivos, que como se mencionó anteriormente, de la eficiencia y equidad en los gobiernos subnacionales. En el caso de México y particularmente Jalisco hay varios estudios relacionados con equidad, pero muy pocos relacionados con la eficiencia. Entre los primeros cabe destacar, Diaz (2006) y Ruiz-Porras y Garcia-Vazquez (2014) entre otros. Sin embargo, hay muy pocos (si es que los hay) estudios a nivel municipal sobre la eficiencia y su relación con las transferencias. Así Avila y Cardenas (2012) que estudian la relación entre la eficiencia relativa de los estados mexicanos y las transferencias, argumentan que las transferencias "etiquetadas" hacen menos eficiente el ejercicio de los recursos debido al menor margen de maniobra presupuestal.

Bajo este contexto, nosotros planteamos la siguiente pregunta: ¿Cuál ha sido la relación entre las transferencias y la eficiencia relativa ${ }^{3}$ en los municipios del estado de Jalisco? Partiendo del supuesto de que una de las funciones de las transferencias intergubernamentales es incrementar la eficiencia de los gobiernos subnacionales, nosotros suponemos que existe una relación inversa entre la cantidad de recursos transferidos y la eficiencia de los municipios. Esto es, los municipios eficientes tendrán un menor monto de transferencias intergubernamentales que los municipios menos eficientes.

Para probar esta hipótesis usamos dos momentos en el análisis. Primero, calculamos las eficiencias relativas de los municipios de Jalisco para los años 1990, 2000 y 2010, a través de la metodología de análisis envolvente de datos o DEA (Data Envelopment Analysis). El segundo momento, usamos los resultados de DEA para identificar los municipios eficientes de los ineficientes y comparamos sus promedios de transferencias (asignaciones y aportaciones) per cápita para los años en cuestión. Encontramos que no hay relación entre

\footnotetext{
${ }^{3}$ La eficiencia relativa de un municipio se entenderá como el nivel de eficiencia de dicho municipio comparado con los otros municipios analizados. Definíéndose eficiencia como la productividad de los recursos utilizados, es decir, la cantidad de recursos necesarios para producir un bien (Bonnefoy y Armijo, 2005)
} 
el monto per cápita promedio de las trasferencias asignadas a los municipios eficientes de los ineficientes. Adicionalmente, detectamos una tendencia incremento en los ingresos municipales no tributarios.

El artículo está organizado como sigue. En la siguiente sección damos una breve revisión de la literatura referente a los estudios realizados con DEA y formulamos la hipótesis que guiará esta investigación. Posteriormente, se describe la metodología del DEA así como sus supuestos además de la descripción de los datos y definición de variables. Luego, realizamos la comparación de los promedios de las transferencias per cápita de los municipios eficientes con relación a los ineficientes. Finalmente, presentamos la discusión de hallazgos y su importancia para direccionar políticas públicas, limitaciones del estudio y oportunidades para futuras investigaciones sobre el tema.

\section{Revisión de la literatura}

A partir de la década de 1990 varios estudios se han realizado con la finalidad de medir la eficiencia de los gobiernos locales o municipales. Por ejemplo, Št’astná y Gregor (2011) mencionan una amplia bibliografía sobre estudios realizados en Europa, Canada y Estados Unidos. También estudios de este tipo se han realizado en Latinoamérica. Estos incluyen los trabajos de Horst (2007) que evalúa la gestión municipal de 45 municipios Chilenos; Sousa y Stošić (2005) estudian los municipios brasileños. Finalmente, Herrera y Francke (2007) analizan los municipios peruanos.

Entre las aportaciones relevantes de estos estudios destacan las siguientes. La mayoría de los municipios pueden mejorar su eficiencia sin incrementar sus gastos corrientes (Afonso y Fernandes, 2008) en especial los más pequeños (Sousa y Stošić, 2005) ya que pueden mejorar significativamente más su eficiencia que los grandes (Št’astná y Gregor, 2011). Sin embargo, los recursos transferidos tienen un impacto negativo en la eficiencia de gobiernos subnacionales (Herrera y Francke, 2007) porque desincentivan recaudar mayores ingresos e incrementan el gasto corriente (Sampere y Sobarso, 1988) y la restringida maniobra en la asignación de los recursos (Ávila y Cárdenas, 2012). Especialmente este fenómeno se da en los municipios pequeños y con un mayor grado de marginación (Noguez, 2006).

En el contexto mexicano, hay muy pocos trabajos, si es que los hay, sobre la eficiencia relativa de los municipios y las transferencias. Así, Ávila y Cárdenas (2012) analizan el impacto de las transferencias condicionadas y la eficiencia técnica de las entidades federativas mexicanas. Estos autores argumentan 
que, como se mencionó anteriormente, existe una ineficiencia de las entidades federativas en el ejercicio de los recursos condicionados provenientes de la federación debido a que tienen un menor margen de maniobra presupuestal.

Así pues, en el estudio de la eficiencia relativa de los gobiernos subnacionales y su asociación con las transferencias intergubenamentales, nos planteamos la interrogante sobre la relación entre las transferencias intergubernamentales ${ }^{4}$ y su asociación con la eficiencia relativa en los municipios de estado de Jalisco. De acuerdo a la Teoría del Federalismo Fiscal, los recursos transferidos a los gobiernos subnacionales tienen como objetivo incrementar la eficiencia en la producción y distribución de bienes y servicios así como la búsqueda de la equidad entre sus gobernados. Con base en este argumento nosotros planteamos la siguiente hipótesis:

Existe una relación inversa entre la eficiencia relativa y los recursos transferidos. En otras palabras, nosotros esperamos que los municipios eficientes tengan un menor monto de transferencias que los ineficientes.

Con la finalidad de probar el argumento anterior usamos dos momentos en el análisis. En el primero, calculamos los índices de eficiencia relativa para los municipios de Jalisco para los años 1990, 2000 y 2010. En el segundo, comparamos las transferencias per cápita de los municipios eficientes con los ineficientes, como a continuación se describe.

\section{Metodología de análisis envolvente de datos}

El análisis envolvente de datos o DEA (Data Envelopment Analysis) es una técnica no paramétrica que consiste en determinar "fronteras" de producción eficientes, en lugar de medidas de tendencia central. Con esta técnica se calcula las relaciones eficientes relativas (scores) a esas fronteras de la Decisión Making Units (DMUs) o municipios a partir del uso de insumos (inputs) y productos (outputs). Esta técnica no paramétrica fue introducida por Charnes, Cooper, y Rhodes $(1978,1981)$ quienes se basaron en la idea de Farrell (1957) sobre la medida de eficiencia a través de un insumo y un producto, para generalizar el análisis a varios insumos y productos. DEA ${ }^{5}$ asume la existencia de una frontera convexa

\footnotetext{
${ }^{4}$ Las transferencias intergubernamentales, también llamados ingresos transferidos (Cabrera y Lozano, 2010), incluyen las participaciones y aportaciones federales.

${ }^{5}$ El término "envolvente" o "envelopment" significa que la frontera de producción envuelve el conjunto de observaciones.
} 
de producción la cual es determinada a través de la programación lineal matemática. Esta técnica no paramétrica nos permite calcular eficiencia técnica relativa de las unidades de decisión (i.e., los municipios) a través de la asignación de valores (scores). De acuerdo con Afonso y Fernandes (2008) la relación general esperada de la eficiencia para cada municipio $i$ es expresada por la siguiente función:

$$
Y_{i}=f\left(X_{i}\right), \quad i=1, \ldots, n
$$

Donde $Y_{i}$ son los indicadores que reflejan las cantidades de productos y $X_{i}$ representa la cantidad de insumos para cada municipio $i$. Estas cantidades pueden ser, según los autores, per cápita o en alguna otra medida. Si $Y_{i}<f\left(X_{i}\right)$ indica que en municipio $i$ es ineficiente, es decir, dado una cantidad de insumos el producto es menor que el mejor nivel posible. Esta ineficiencia relativa del municipio $i$ que está debajo de la frontera "envolvente," la cual es calculada (no estimada) por la desviación de la frontera. Esto es, entre más alejadas estén las relaciones insumos-productos de los municipios de la frontera más ineficientes serán. ${ }^{6}$

Charnes, Cooper, y Rhodes (1981) señalan que hay dos maneras de construir la frontera envolvente para las Decision Making Units (DMUs): a) orientación en los productos u b) orientación en los insumos. En el primero se argumenta que un municipio es eficiente si no es posible aumentar la cantidad de producto si aumentar los insumos o reducir la producción de otro producto. El segundo caso, un municipio es eficiente si no es posible reducir la cantidad de insumos para producir la cantidad de productos establecida. Los dos métodos proporcionan los mismos resultados bajo el supuesto de rendimientos constantes a escala pero dan diferentes valores cuando los rendimientos son variables (Afonso y Fernandes, 2008). Sin embargo, de acuerdo a estos autores, ambos modelos identificaran el mismo conjunto de eficientes/ineficientes DMUs. Finalmente, los valores de eficiencia relativa serán uno para los eficientes (i.e., están en la frontera) y menos de uno para los ineficientes (i.e., no están en la frontera).

\footnotetext{
${ }^{6}$ Ver, por ejemplo, Murillo-Zamorano (2004) donde se presenta una discusión interesante sobre este tipo de modelos.
} 


\section{Supuestos del Modelo DEA}

Nosotros usamos DEA con insumo-orientada (i.e., minimizar inputs para obtener la misma cantidad de outputs) con rendimientos variables a escala (i.e., si se duplica los insumos se espera menos del doble de outputs) para calcular la eficiencia técnica general. Esto es, nos enfocamos en la reducción de los insumos (gasto corriente per cápita) de los municipios. Esta orientación es adoptada porque se asume que los municipios consideran los productos (servicios prestados) como exógenos o independientes y además, tienen control sobre los insumos (Worthington y Dollery, 2001). Los municipios mexicanos y particularmente los de Jalisco, ya tienen una demanda constante de bienes y servicios, por ejemplo, el alumbrado público. De aquí que, los municipios tiendan a minimizar los insumos en lugar de maximizar los productos. Por lo tanto, el cálculo de la eficiencia técnica orientada sobre el o los insumos nos permite detectar fallas en el uso eficiente de los mismos. Comparado con otros municipios similares que logran satisfacer la demanda del servicio con menos recursos. El supuesto de rendimientos constantes no fue considerado, porque no se espera que cuando se duplican los insumos se duplique los productos. Por ejemplo, si duplicamos los recursos para el alumbrado público, en un determinado municipio, no esperamos que el municipio duplique el alumbrado público. Aunque se espera un incremento o calidad en dicho servicio. A continuación se definen las unidades de decisión (DMUS), insumos (inputs) y productos (outputs).

\section{Unidades de decision (DMUS)}

Lo municipios del estado de Jalisco fueron considerados como las unidades de decisión (DMUs). Los municipios considerados fueron todos aquellos que reportaron datos sobre sus finanzas públicas y población durante las décadas de 1990, 2000, y 2010, según el Instituto Nacional de Estadística y Geografía (INEGI) ${ }^{7}$ y el Sistema Municipal de Base de Datos (SIMBAD). ${ }^{8}$ Los municipios seleccionados fueron todos aquellos que reportaron datos sobre sus insumos, productos y transferencias. Por ejemplo, para el año 1990 no se consideró Guadalajara y otros 23 municipios porque no encontró datos sobre "servicios generales". Usamos el índice de precios reportado por el Banco de México para deflactar los valores de

\footnotetext{
7 http://www.inegi.org.mx/

${ }^{8}$ Sistema Municipal de Base de Datos (SIMBAD) en http://sc.inegi.org.mx/sistemas/cobdem/contenido-arbol.jsp?rf=false Accesado el 4 de mayo de 2015.
} 
las variables tomando 2010 como año base. Los indicadores ${ }^{9}$ considerados están expresados per cápita y a precios contantes del 2010.

\section{Insumos (Inputs)}

El gasto corriente per cápita para cada municipio fue considerado como insumo (input). El gasto corriente (i.e., aquel que no aumenta los activos del gobierno) que más adelante se define como la suma de todos los costos de insumos y servicios (input) para que los ayuntamientos puedan cumplir con sus obligaciones establecidas en la Constitución. Este criterio ha sido utilizado ampliamente en la literatura, por ejemplo, ver Afonso y Fernandes (2008) donde se muestran varios estudios donde se usa el gasto corriente como insumo. En este estudio, consideramos el gasto corriente en forma desagregada. Esto es, servicios personales ${ }^{10}$ (SP), materiales y suministros ${ }^{11}$ (MYS) y servicios generales ${ }^{12}$ (SG). En el cuadro 1 se presenta las estadísticas básicas de estas variables así como el cambio porcentual entre décadas. Observamos que el promedio de los servicios personales (SP) en los municipios jaliscienses tuvieron un aumento sostenido en los años considerados, pasan de 193 pesos per cápita promedio en 1990 a 1244 en 2010. Esto es, un incremento de aproximadamente $192 \%$ para $1990-2000$ y un incremento menor para 2000 2010 de 120\%. Los materiales y suministros (MYs) tuvieron una caída de $28 \%$ para la primera década pero un incremento de $88 \%$ en la segunda década. Finalmente, el cuadro 1 nos muestra que los servicios generales tuvieron una disminución importante pues pasaron de $285 \%$ en el primer periodo al solo un incremento de $22 \%$ para el segundo periodo.

\footnotetext{
${ }^{9}$ Para medir el desempeño gubernamental, tarea bastante compleja, hay básicamente dos tipos de medidas relativas (i.e., indicadores tales como tasa de natalidad, gasto corriente per cápita, entre otros) y absolutas (i.e., medidas físicas tales como número de empleados, tamaño de oficinas, escuelas construidas, entre otros). Se argumenta en la literatura que las medidas relativas minimizan las diferencias de calidad, por ejemplo, de bienes y servicios provistos por los gobiernos en estudio (Ávila y Cárdenas, 2012).

${ }^{10}$ Remuneraciones al personal que presta sus servicios al gobierno así como cualquier otra erogación derivada de la relación laboral (INEGI, 2009).

${ }^{11}$ Costo de toda clase de materiales requeridos por los gobiernos para el desempeño de sus actividades administrativas y productivas (INEGI, 2009).

${ }^{12}$ Costo de todos los servicios indispensables para el funcionamiento de la administración pública incluye teléfono, luz eléctrica, agua potable, mantenimiento, entre otros (INEGI, 2009).
} 


\section{Cuadro I}

Estadística Descriptiva de Insumos para los Diferentes Años

\begin{tabular}{lrrrrrrrrrr} 
& \multicolumn{3}{c}{ Servicios Personales } & \multicolumn{3}{c}{$\begin{array}{c}\text { Materiales } \\
\text { y Suministros }\end{array}$} \\
& 1990 & 2000 & 2010 & 1990 & 2000 & 2010 & 1990 & 2000 & 2010 \\
Media & 193 & 565 & 1244 & 311 & 224 & 422 & 123 & 475 & 579 \\
DE & 127 & 208 & 378 & 197 & 177 & 259 & 99 & 211 & 233 \\
Mínimo & 31 & 148 & 643 & 5 & 10 & 89 & 0 & 42 & 236 \\
Máximo & 862 & 1108 & 2734 & 1106 & 1470 & 1419 & 612 & 1397 & 1357 \\
Cambio Porcentual & & 193 & 120 & & -28 & 88 & & 285 & 22 \\
\hline
\end{tabular}

Elaboración propia. Datos a precios constantes de 2010 (Banco de México) y per cápita. $\mathrm{DE}=$ Desviación estándar.

Productos (outputs)

Usamos dos productos (outputs), la recolecta de los ingresos propios y el gasto en obra social o inversión en capital. Básicamente por dos razones. Primera, en el Artículo 115 de la constitución mexicana, se establecen las responsabilidades de los municipios, por ejemplo, alumbrado público, calles, parques y jardines; cuyos gastos están reportados en el rubro de gasto en obra social. Adicionalmente, en el citado artículo constitucional se menciona que los municipios tienen la capacidad de establecer y recolectar impuestos, por ejemplo, el predial entre otros. Segunda, debido a la dificultad de medir los productos (resultados) de los municipios y obtener información estadística de ellos o parte de ellos, decidimos utilizar los egresos reportados en el rubro de gasto en obra social y los montos de los ingresos reportados por el SIMBAD ${ }^{13}$ del INEGI.

Para realizar el análisis usamos tres productos: la inversión pública ${ }^{14}$ (IP), ingresos tributarios (IT) e ingresos no tributarios (INT). Donde IP incluye obra

\footnotetext{
${ }^{13}$ Sistema Estatal y Municipal de Bases de Datos (SIMBAD), estadísticas de finanzas públicas estatales y municipales.

${ }^{14}$ A partir del 2012 la partida de "obra pública y acciones sociales" se reclasifico como "inversión pública”. De acuerdo al INEGI datos correspondientes a 1989-2012 ya se encuentra homologada con estos criterios. Para mayor detalle consultar la página: http://sc.inegi.org.mx/cobdem/resultados.js $\mathrm{p} ? \mathrm{w}=12 \&$ Backidhecho $=108 \&$ Backconstem $=106 \&$ constembd $=038 \& \mathrm{tm}={ }^{\prime}$ Backidhecho: 3 , Backco nstem:3, constembd:3' Accesado el 4 de mayo de 2015.
} 
pública $^{15}$ y acciones sociales; iT es la suma de impuestos ${ }^{16}$ y derechos; y INT es la suma de productos ${ }^{17}$ y aprovechamientos. A continuación en el cuadro 2 se muestra que IP tuvo un incremento sostenido paso de 238 pesos per cápita en 1990 a 981 en el 2010. El cambio porcentual en este rubro durante el periodo $1990-2000$ fue de $203 \%$ y solo un 36\% para el segundo periodo. Con respecto a los ingresos tributarios, muestran un patrón similar. De 1990 a 2000 se pasó de 143 pesos per cápita a 309 pesos lo que nos indica un crecimiento de 116\%. Pero para el periodo 2000-2010 el cambio solo fue de 32\%. Finalmente, los ingresos no tributarios mostraron un patrón diferente. Pasó de $35 \%$ en el primer periodo a un incremento importante para el segundo periodo de $147 \%$. Se observa en el mismo cuadro 2 que los municipios del estado de Jalisco, los ingresos no tributarios per cápita (\$590) en el año 2010 son mayores que los ingresos tributarios per cápita $(\$ 408)$ en el mismo año.

\section{Cuadro 2}

\section{Estadística descriptiva de productos para los diferentes años}

\begin{tabular}{lrrrrrrrrr} 
& \multicolumn{3}{c}{ Inversión Pública } & \multicolumn{3}{c}{ Ingresos Tributarios } & \multicolumn{4}{c}{ No Tributarios } \\
& \multicolumn{1}{c}{} & & & & & \multicolumn{5}{c}{ No Tributar } \\
& 1990 & 2000 & 2010 & 1990 & 2000 & 2010 & 1990 & 2000 & 2010 \\
Media & 238 & 720 & 981 & 143 & 309 & 408 & 177 & 239 & 590 \\
DE & 232 & 561 & 1065 & 148 & 177 & 189 & 253 & 298 & 849 \\
Mínimo & 13 & 52 & 83 & 18 & 8 & 96 & 6 & 9 & 28 \\
Máximo & 1329 & 3190 & 6664 & 1268 & 1107 & 1139 & 1583 & 1962 & 6148 \\
Cambio Porcentual & & 203 & 36 & & 116 & 32 & & 35 & 147 \\
\hline
\end{tabular}

Elaboración propia. Datos a precios constantes de 2010 (Banco de México) y per cápita. $\mathrm{DE}=$ Desviación estándar.

\footnotetext{
${ }^{15}$ De acuerdo al INEGI (2009), los recursos destinados a la construcción de obras públicas (por ejemplo, carreteras, puentes, etc.) contribuye a la formación de capital del país (INEGI, 2009) y los recursos destinados a las acciones sociales contribuye con el bienestar social de la población, por ejemplo, la seguridad pública.

${ }^{16}$ Contribuciones que el gobierno fija unilateralmente. Por ejemplo, el impuesto de $2 \%$ sobre nómina, predial y sobre transmisión de dominio; los derechos son contribuciones establecidas por ley por el uso o aprovechamiento de los bienes de dominio público, por ejemplo, el registro civil, licencias y permisos (INEGI, 2009).

${ }^{17}$ Los productos son ingresos que no corresponden al desarrollo de las funciones propias del derecho público, sino por la explotación de sus bienes patrimoniales tales como enajenación de bienes muebles e inmuebles, arrendamientos e intereses; los aprovechamientos se originan por el incumplimiento de las contribuciones fiscales de los contribuyentes y que son recibidos en forma de rezagos, recargos, reintegros, donativos y multas (INEGI, 2009).
} 


\section{Transferencias Intergubernamentales}

Las transferencias intergubernamentales (TR) o transferencias federales, también llamados ingresos transferidos (Cabrera y Lozano, 2010), incluyen las Participaciones ${ }^{18}$ o Ramo 28 que tienen una finalidad resarcitoria y las Aportaciones $^{19}$ o Ramo 33 con objetivos compensatorios. En general se definen las transferencias como "los recursos transferidos de un orden de gobierno a otro con el fin de financiar las necesidades de gasto del ámbito receptor" (Peña, 2012, 13). De acuerdo a Peña (2012), las transferencias federales como él se refiere a las transferencias intergubernamentales cumplen dos funciones básicas e incluso ambas a la vez. Una reducir la brecha entre los ingresos propios y las necesidades de gasto (i.e., disminuir el desequilibrio vertical) o reducir la brecha entre la capacidad de gasto entre gobiernos de un mismo nivel (i.e., disminuir el desequilibrio horizontal) este argumento concuerda con y Ahmad y Craig (1997). En este estudio consideramos a los ingresos transferidos como la suma de las participaciones y aportaciones recibidas por los gobiernos municipales del estado de Jalisco. Así mismo en este estudio no se discutirán los mecanismos de asignación sino más bien la relación entre estos y la eficiencia relativa de los municipios jaliscienses para los años 1990, 2000, y 2010.

En el cuadro 3 observamos un incremento constante de las transferencias en los municipios del estado de Jalisco durante los periodos de 1990-2000 y 2000-2010. Pasaron de 628 pesos per cápita en 1990 hasta 2627 pesos per cápita en el 2010. Sin embargo, el crecimiento fue mayor en el primer periodo $196 \%$ contra un crecimiento de $41 \%$ en el periodo $2000-2010$.

\footnotetext{
18 "Asignaciones de recursos fiscales que otorga el Gobierno Federal a los Estados, Municipios y Distrito Federal, a través de fondos destinados a cubrir la parte de los ingresos federales que recaudan las oficinas receptoras y que les corresponden de acuerdo con las disposiciones legales" (INEGI, 2009 , p. 6).

19 "Son los recursos que otorga la Administración Pública Federal a los Gobiernos de los Estados, Municipios y Distrito Federal, destinados a cubrir gastos de los objetivos y programas delegados a estos dos niveles de gobierno. Se canalizan a través del Ramo General 33, Ramo 25, Convenios descentralizados y Convenios reasignados" (INEGI, 2009, p. 6).
} 
Economía Informa núm. 393 julio - agosto • 2015

\section{Cuadro 3}

Estadística Descriptiva de las Transferencias

\begin{tabular}{lrrr} 
& \multicolumn{3}{c}{ Transferencias } \\
& 1990 & 2000 & 2010 \\
Media & 628 & 1857 & 2627 \\
DE & 292 & 815 & 1333 \\
Mínimo & 67 & 149 & 787 \\
Máximo & 2033 & 4542 & 7894 \\
Cambio Porcentual & & 196 & 41 \\
\hline
\end{tabular}

Elaboración propia. Datos a precios constantes de 2010

(Banco de México) y per cápita. DE=Desviación estándar.

En la Grafica 1 presentamos la relación entre el monto de los recursos transferidos a los municipios del estado de Jalisco en los años 1990, 2000 y 2010 con el tamaño de los municipios (ver Anexo, Tabla 4 con la descripción del número de municipios por factor de clasificación). Se puede notar en la Grafica 1 que se incrementó las TR per cápita durante los años considerados. Pero hubo una relación inversa entre TR y el tamaño del municipio. Esto es, los municipios con mayor población recibieron menos recursos transferidos que los municipios con menos población (i.e., los ubicados en el primer cuartil en términos de su número de habitantes). Este comportamiento fue similar en los años considerados. 


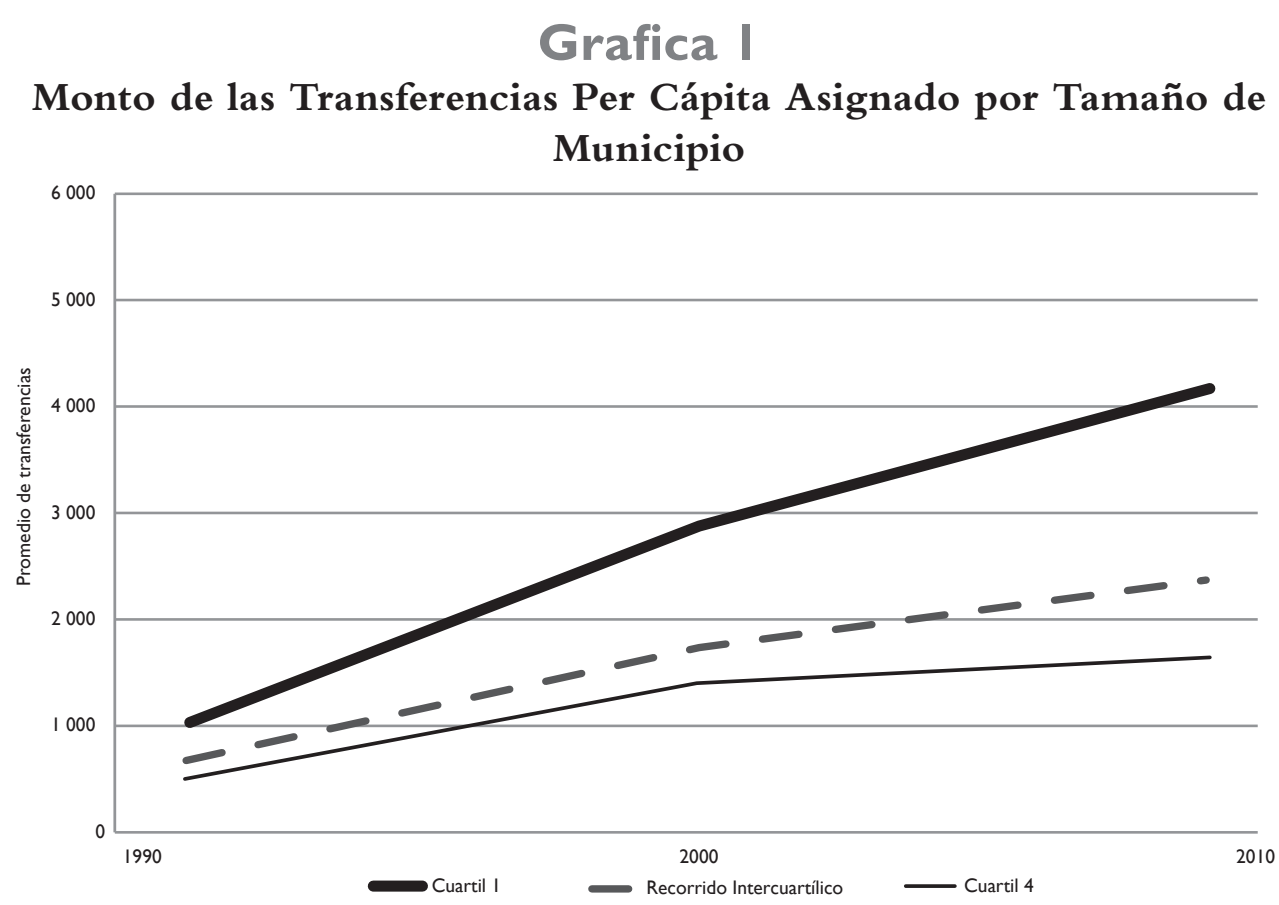

Fuente: elaboración propia.

En suma, de los cuadros 1,2 y 3 y la grafica 1 podemos concluir lo siguiente. Hubo un incremento de los servicios personales, servicios generales, inversión pública, los ingresos tributarios y las transferencias en los municipios del estado de Jalisco durante los periodos 1990-2000 y 2000-2010. Pero el crecimiento fue menor en el segundo periodo de 10 años comparado con el primero. Sin embargo, hubo un crecimiento mayor en el segundo periodo comparado con el primero con los materiales y suministros y con los ingresos no tributarios. En grafica 1, observamos la relación inversa entre los montos de recursos transferidos per cápita y tamaño en términos de población.

\section{Estudio en dos etapas}

El estudio para probar la hipótesis planteada sobre la relación inversa entre las transferencias y la eficiencia relativa en los municipios jaliscienses durante los años 1990, 2000 y 2010 es presentado en dos etapas. En la primera etapa, los municipios seleccionados anteriormente se calculó su respetivo índice de eficiencia relativa a través del análisis envolventemente de datos (DEA). Posteriormente se compara las transferencias per cápita de los muni- 
cipios eficientes con los municipios ineficientes. Los índices de eficiencia se calcularon con el programa de cómputo Banxia Frontier Analyst 2.0.

\section{Primera etapa}

La clasificación de los municipios se realizó en cuartiles con base a su población para los años en cuestión. Esta clasificación se hizo para obtener unidades de decisión (los municipios) lo más homogéneas posible. Por ejemplo, para 1990 solo hay reporte de información en el SIMBAD para 100 municipios. Donde el rango de la población va de 1001021 (Zapopan) a 2155 (Ejutla) habitantes. El primer cuartil incluye 25 municipios cuya población va de 2155 a 6382 , el cuarto cuartil incluye también 25 municipios cuya población va de 34329 a 1001021 habitantes. Finalmente, el recorrido inter-cuartilico que incluye 50 municipios cuya población está entre 6533 a 34329 habitantes. Este mismo procedimiento se aplicó para los municipios en los años 2000 y 2010.

Los resultados del análisis DEA en el cuadro 4 nos indican que en la mayoría de los municipios del cuarto cuartil (grandes) fueron eficientes en los años considerados. Sin embargo, los municipios medianos, es decir, los ubicados en el recorrido inter-cuartílico la mayoría resulto ser ineficiente. Para los municipios ubicados en el primer cuartil (pequeños) los resultados son mixtos. Se observa que en los años 1990 y 2000 la mayoría fue eficiente. Pero en el año 2010 el número de municipios ineficientes se incrementó a 18.

Adicionalmente, en el cuadro 4 tenemos datos que nos indican los porcentajes de insumos que se pueden reducir los municipios ineficientes para alcanzar el nivel de producto de los municipios eficientes. Por ejemplo, para el año 2010 los municipios del cuarto cuartil pudieron reducir sus servicios personales (salarios) en $8 \%$, los materiales y suministros en $11.6 \%$ y los servicios generales en $8.8 \%$ para incrementar, en promedio, $21.4 \%$ en inversión pública, $14.5 \%$ sus ingresos tributarios y un $35.7 \%$ sus ingresos no tributarios. En este cuartil también podemos observar que la potencialidad de captación de ingresos propios vino decreciendo desde 1990 a 2010, ya que pasó de 30\% en 1990 hasta $14.5 \%$ en el 2010. Sin embargo, el comportamiento con los ingresos no tributarios ha tenido un comportamiento inverso, ya que paso de 8.4\% en 1990 a 35.7\% para el 2010. Para los municipios que están en el primer cuartil (pequeños) los ingresos tributarios y no tributarios tienen un comportamiento creciente con una mayor intensidad los ingresos no tributarios. Para $50 \%$ de los municipios centrales los resultados son mixtos pero se nota un alto 
porcentaje para mejorar, por parte de los municipios ineficientes, en el 2010 de cerca $63 \%$ en cuanto a la captación de ingresos no tributarios.

En suma, podemos argumentar que los resultados del cuadro 4 nos sugieren que los municipios del estado de Jalisco eficientes han mejorado en mucho en cuanto a su captación de ingresos no tributarios y en la captación de ingresos tributarios. Hay un amplio margen de captar ingresos no tributarios en todos los municipios, acompañado de una reducción menor en la cantidad de sus insumos.

\section{Cuadro 4 \\ Resultado del análisis DEA}

\begin{tabular}{|c|c|c|c|c|c|c|c|c|c|c|}
\hline $\begin{array}{c}\text { Cuartil } \\
\text { (núm. de } \\
\text { habitantes) }\end{array}$ & Año & $\mathrm{N}$ & Eficientes & Cuarto Cuar & $\mathrm{SP} \%$ & MYs $\%$ & $\mathrm{SG} \%$ & $\mathrm{IP} \%$ & $\mathrm{IT} \%$ & INT $\%$ \\
\hline $1001021-35432$ & 1990 & 25 & 14 & 11 & -11.0 & -17.0 & -13.7 & 20.0 & 30.0 & 8.4 \\
\hline 1646 319-35 502 & 2000 & 21 & 12 & 9 & -10.5 & -14.4 & -10.5 & 13.5 & 28.7 & 22.3 \\
\hline 1495 189-34 829 & 2010 & 31 & 18 & 13 & -8.0 & -11.6 & -8.8 & 21.4 & 14.5 & 35.7 \\
\hline \multicolumn{11}{|c|}{ Recorrido Inter-Cuartilico } \\
\hline $34329-6533$ & 1990 & 50 & 14 & 36 & -8.3 & -9.3 & -9.2 & 51.3 & 0.5 & 21.4 \\
\hline $35432-6974$ & 2000 & 40 & 19 & 21 & -12.3 & -16.3 & -12.8 & 28.7 & 13.2 & 16.7 \\
\hline $34182-6685$ & 2010 & 62 & 15 & 47 & -3.8 & -6.8 & -3.9 & 19.9 & 2.8 & 62.9 \\
\hline \multicolumn{11}{|c|}{ Primer Cuartil } \\
\hline $6384-2155$ & 1990 & 25 & 14 & 11 & -6.14 & -8.03 & -6.57 & 59.57 & 1.37 & 18.33 \\
\hline $6577-2155$ & 2000 & 21 & 11 & 10 & -20.6 & -19.2 & -21.2 & 14.1 & 4.4 & 20.4 \\
\hline 6 655-2 082 & 2010 & 31 & 13 & 18 & -7.9 & -10.3 & -9.8 & 7.5 & 7.9 & 56.7 \\
\hline
\end{tabular}

Elaboración propia partir de los resultados de DEA.

\section{Segunda etapa}

En el análisis de la segunda etapa comparamos las transferencias intergubernamentales per cápita de los municipios eficientes de los ineficientes. Los resultados sugieren que no hay una diferencia estadística significativa entre los promedios de transferencias intergubernamentales per cápita de los municipios eficientes de los ineficientes. Estos resultados fueron consistentes tanto en forma global como para los grupos cuartilicos y para los años considerados. Por ejemplo, en el cuadro 5 podemos notar que para el grupo de municipios 
del cuarto cuartil y para el año 2000 se muestra una diferencia de -16 (el monto de transferencias per cápita de los municipios eficientes fue menor que sus contrapartes), sin embargo, esta diferencia no fue estadísticamente significativa. Esta prueba fue realizada asumiendo varianzas homogéneas como lo indica la prueba de Levene.

\section{Cuadro 5}

\section{Diferencia de medias de las Transferencias Intergubernamentales de los municipios eficientes e ineficientes}

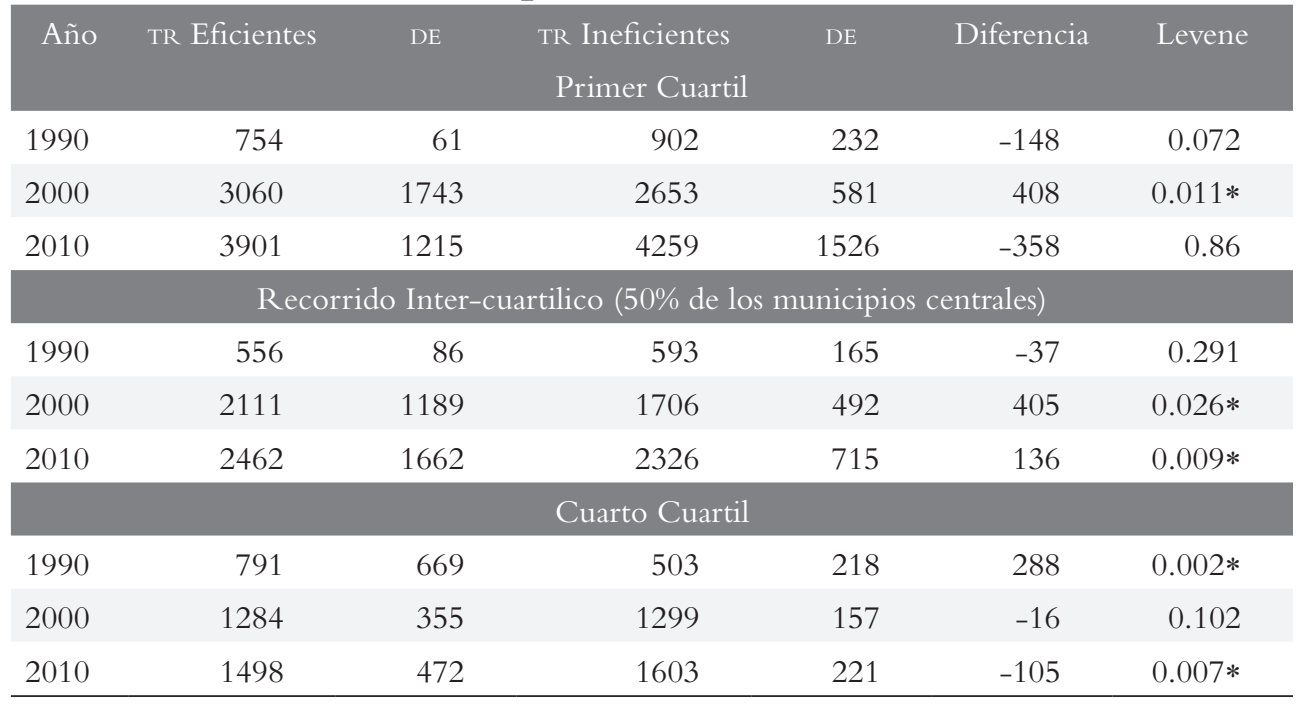

Notas: la diferencia de TR entre los municipios eficientes y no eficientes no fue estadísticamente significativa para ningún grupo y año. *El valor del estadístico de Levene representa el valor de significancia, es decir, el valor $\mathrm{p}$. Para valor $\mathrm{p}<0.05$ se realizó la prueba de medias asumiendo varianzas no homogéneas. DE $=$ Desviación Estándar.

En general, los resultados del cuadro 5 nos indican que para los municipios del estado de Jalisco en los años de 1990, 2000 y 2010 no hubo relación entre su eficiencia relativa y las trasferencias intergubernamentales. Adicionalmente, se presenta los resultados del análisis de varianza (ANOVA) de tres factores (i.e., año, cuartil y eficiencia) donde se hacen las pruebas estadísticas conjuntas. En el cuadro 6 se muestran los municipios clasificados de acuerdo a los factores considerados. Por ejemplo, en el año 1990 solo se consideraron 100 del total de municipios del Estado; para el 2000 fueron 82 y para el 2010 fueron 124 que nos da un total de 306. En los tres periodos del análisis de estos 306 solo 175 reportaron un puntaje de eficiencia relativa de $100 \%$ mientras que el resto de los municipios su eficiencia fue menor. 


\section{Cuadro 6}

\section{Descripción de los Municipios Jaliscienses \\ Considerados en el Estudio}

\begin{tabular}{|c|c|c|c|}
\hline & & Característica & $\mathrm{N}$ \\
\hline \multirow{3}{*}{ Año } & 1 & 1990 & 100 \\
\hline & 2 & 2000 & 82 \\
\hline & 3 & 2010 & 124 \\
\hline \multirow{3}{*}{ Cuartil } & 1 & Cuartil 1 & 77 \\
\hline & 2 & Recorrido Intercuartilico & 152 \\
\hline & 3 & Cuartil 4 & 77 \\
\hline \multirow{2}{*}{ Eficiencia } & 0 & No eficiente & 175 \\
\hline & 1 & Eficiente & 131 \\
\hline
\end{tabular}

En el cuadro 7 se muestran los resultados de ANOVA de tres factores asociados con la variable dependiente transferencias per cápita. Podemos observar que la diferencia de transferencias per cápita entre los municipios eficientes e ineficientes no es estadísticamente significativa $(p<0.246)$. Igualmente no hubo diferencia estadística cuando se consideraron por grupo y cuando se consideró conjuntamente por año y grupo. En este caso, tenemos que la variación de las transferencias per cápita de los municipios considerados está explicada en promedio $73 \%$ por los factores considerados, es decir, por año, grupo y eficiencia. 


\section{Cuadro 7}

\section{Resultados de ANOvA con tres vías}

\begin{tabular}{|c|c|c|c|c|c|}
\hline \multicolumn{6}{|c|}{ Variable Dependiente:Transferencias Per Cápita } \\
\hline Fuente & $\begin{array}{l}\text { Tipo III Suma de } \\
\text { Cuadrados }\end{array}$ & $\mathrm{gl}$ & $\begin{array}{l}\text { Cuadrados } \\
\text { Medios }\end{array}$ & $\mathrm{F}$ & Sig \\
\hline Modelo Corregido & $372769818.273^{a}$ & 17 & 21927636.369 & 48.476 & .000 \\
\hline Intercepto & 779136026.592 & 1 & 779136026.592 & 1722.470 & .000 \\
\hline Año & 191230101.624 & 2 & 95615050.812 & 211.380 & .000 \\
\hline Cuartil & 94595125.016 & 2 & 47297562.508 & 104.563 & .000 \\
\hline Eficiencia & 610782.063 & 1 & 610782.063 & 1.350 & .246 \\
\hline Año * Cuartil & 30762125.443 & 4 & 7690531.361 & 17.002 & .000 \\
\hline Año * Eficiencia & 3067361.271 & 2 & 1533680.636 & 3.391 & .035 \\
\hline Cuartil * Eficiencia & 260413.037 & 2 & 130206.519 & .288 & .750 \\
\hline Año $*$ Cuartil $*$ Eficiencia & 1392220.527 & 4 & 348055.132 & .769 & .546 \\
\hline Error & 130272884.185 & 288 & 452336.403 & & \\
\hline Total & 1459155926.000 & 306 & & & \\
\hline Corrección Total & 503042702.458 & 305 & & & \\
\hline
\end{tabular}

Con base en los anteriores resultados, contrariamente lo que supusimos, no encontramos asociación estadística entre la eficiencia de los municipios jaliscienses y el monto de recursos transferidos por los diferentes órdenes de gobierno al menos en los años considerados en el estudio.

Sin embargo, como se muestra en los Anexos A, B, y C, hubo mejora en cuanto a su eficiencia relativa. Para los municipios de cuarto cuartil pasaron de $91 \%$ en promedio en 1990 a 96\% en 2010. Los municipios ubicados en el recorrido intercuartilico pasaron de $74 \%$ en 1990 a $89 \%$ en el 2010. Finalmente, los municipios ubicados en el primer cuartil se mantuvieron en $89 \%$ aunque en el 2000 alcanzaron 83\%. Estos resultados junto con la información de la Grafica 1 nos sugiere que hay un relación positiva entre el incremento del monto de las transferencias per cápita y la eficiencia en los municipios del estado de Jalisco.

\section{Conclusiones y recomendaciones}

El objetivo en este artículo es el estudio de la relación entre la eficiencia relativa de los municipios y las transferencias intergubernamentales. El argumento 
basado en la Teoría del Federalismo Fiscal donde se establece la asignación de responsabilidades (e.g., proveer servicios públicos) y facultades (e.g., imponer tributos) a los gobiernos municipales por parte del gobierno central para satisfacer la demanda de bienes y servicios públicos para sus gobernados. Sin embargo, la asignación de estas actividades normalmente genera desequilibrios tanto horizontales (i.e., capacidades fiscales diferentes) como verticales (i.e., capacidad de ingreso y gasto son desiguales en los niveles de gobierno) en las finanzas municipales. Las transferencias o recursos transferidos son un mecanismo para aminorar esta situación. De hecho, se argumenta en la literatura que el objetivo de los recursos trasferidos es la búsqueda de la eficiencia y equidad interjurisdicional. A pesar que también pueden tener su lado obscuro como generar pereza fiscal en los gobiernos municipales.

En este contexto nosotros formulamos la pregunta sobre la relación de la eficiencia relativa de los municipios y el monto de los recursos transferidos. Así mismo, supusimos que había una relación inversa entre el monto de recursos transferidos y la eficiencia relativa. Esto es, los municipios eficientes tendrían un menor monto de transferencias que los ineficientes. Para probar nuestra hipótesis y consecuentemente contestar la pregunta utilizamos información de las finanzas públicas y datos sobre población de municipios jaliscienses para los años 1990, 2000, y 2010. El análisis lo realizamos en dos etapas. Primero calculamos las eficiencias relativas para cada uno de los municipios, previamente agrupados en cuartiles, a través de la metodología del DEA (Data Envelopment Analysis) y posteriormente comparamos los recursos transferidos de los municipios eficientes con los ineficientes.

Los resultados del análisis indican que no hubo relación entre las transferencias y la eficiencia en los municipios. Esto es, para los municipios jaliscienses durante los años 1990, 2000, y 2010 no existió diferencia estadística en los promedios de recursos transferidos per cápita de los municipios eficientes con los ineficientes. A pesar que no se cumplió la hipótesis planteada, podemos argumentar que hubo en incremento en la eficiencia relativa de los municipios jaliscienses. Como se muestra en la Grafica 1 y los resultados en los Anexos A,B y C.

Adicionalmente, a partir del análisis sobre el cálculo de la eficiencia relativa (ver Cuadro 4) se encontró que los municipios jaliscienses han incrementado su captación tributaria y no tributaria. Especialmente, existe potencial en los no tributarios, por ejemplo, en el 2010 de los 62 municipios con una población que va de 34,182 a 6,685 habitantes y con una reducción en sus insumos de entre un 3.8 a 6.8\% pueden incrementar en un 19.9\% la inversión 
pública y sobre todo tienen el potencial para incrementar su base recaudadora en impuestos no tributario en casi 63 por ciento.

En suma, los resultados de este artículo los podemos sintetizar como sigue. En el análisis realizado con los municipios de Jalisco durante los años 1990, 2000 y 2010 encontramos que no existió una relación entre eficiencia y las transferencias intergubernamentales. Sin embargo, se detectó una asociación positiva entre el monto de las transferencias y el incremento en la eficiencia de los municipios. Finalmente, la administración de estos municipios tiene potencial para incrementar la captación de ingresos no tributarios como lo están haciendo los municipios eficientes.

Los claroscuros que presentan la asociación entre los recursos transferidos y la eficiencia se deben principalmente a que no hay incentivos para los municipios ineficientes para cambiar su status quo, por lo que es racional para los municipios mantenerse como ineficientes. De hecho, se argumenta en la literatura que los recursos transferidos a las municipios tienen impacto negativo en la eficiencia del gasto de los recursos además de desincentivar recaudar mayores ingresos (Herrera y Francke, 2007).

Con base en lo anterior se recomienda que haya una mejor coordinación entre los diferentes niveles de gobierno para no ocasionar una mala distribución de los servicios y así evitar altas transferencias federales que pueden incentivar al gasto ineficiente de los gobiernos locales y desincentivar recaudar mayores ingresos (Cabrera y Lozano, 2010). En este mismo sentido, sugieren que en la medida de lo posible los gastos locales deben ser cubiertos con ingresos propios porque se hace más eficiente el gasto (Sampere y Sobarzo, 1988).

El presente estudio, como todos los demás, tiene sus limitaciones. La homogeneidad de las unidades de análisis es básica para el cálculo de la eficiencia. Por ejemplo, en el cuarto cuartil se compara la eficiencia relativa de Teocaltiche $(40,105)$ y Guadalajara (1’469,140 h) que están muy lejos de ser municipios homogéneos. También el hecho de tener mayor gasto per cápita en inversión pública no necesariamente implica que el gasto haya sido eficiente. Por ejemplo, el gasto en pavimentación con mala calidad implicaría que cada temporada de lluvias se tenga que estar renovando. También, hay gastos en infraestructura que no tienen ninguna o muy poca utilidad para la comunidad (por ejemplo, las construcciones llamadas "elefantes blancos"). Otra limitante de este trabajo es el supuesto de considerar los mismos costos en la producción y provisión de productos o servicios en los municipios. Por ejemplo, la construcción de una carretera en una zona montañosa es diferente que el de una zona plana. Adicionalmente, los municipios más pequeños tienden 
a tener costos promedio más altos en la producción de bienes y servicios que los municipios grandes debido a las economías de escala. Esto sugiere que los resultados aquí presentados tienen que ser interpretados con precaución.

Sin embargo, los resultados de eficiencia en este análisis no incluyen la equidad que es una parte complementaria del desempeño público. Esto es, si un municipio es eficiente no necesariamente implica que sea equitativo. Por ejemplo, un municipio eficiente puede no dar el mismo trato a sus ciudadanos en situaciones similares (equidad horizontal) o garantizar la distribución igualitaria en el ingreso (equidad vertical).

Este estudio contribuye a la literatura sobre la eficiencia relativa de los gobiernos locales especialmente mexicanos donde hay muy pocas investigaciones. De hecho, casi no hay trabajos donde se aborde el análisis de la eficiencia municipal a través del gasto corriente, es decir, servicios personales, materiales y suministros y servicios generales como insumos y como productos la inversión pública y la captación de impuestos tributarios y no tributarios. Así mismo, este estudio proporciona los resultados de eficiencia que obtuvieron los diferentes municipios jaliscienses y potencialidad de mejora comparado con los que un tuvieron un mejor desempeño.

Para futuras investigaciones dentro de estos temas debe considerarse atenuar el problema de la homogeneidad. Pues ante la falta de información municipal completa en todas sus variables, existió una gran heterogeneidad entre el comportamiento de los municipios que dificultó su comparación. Esto se facilitaría si se tuviera grupos con unidades de análisis más homogéneos, por ejemplo, considerando un estudio para los municipios de la Región Occidente de México o del país en general. Finalmente, debido a la falta de espacio es imposible mostrar en este documento los resultados del análisis del DEA para cada municipio incluido en el estudio. Los resultados en detalle pueden ser obtenidos de los autores sobre pedido. 
Economía Informa núm. 393 julio - agosto • 2015

\section{Anexo A}

Cuadro con el puntaje de eficiencia relativa de los municipios del cuarto cuartil.

\begin{tabular}{|c|c|c|c|c|c|}
\hline \multicolumn{6}{|c|}{ Cuarto Cuartil } \\
\hline \multicolumn{2}{|l|}{1990} & \multicolumn{2}{|l|}{2000} & \multicolumn{2}{|l|}{2010} \\
\hline Unit & Score & Unit & Score & Unit & Score \\
\hline Teocaltiche & 100 & Tamazula de Gordiano & 100 & Tlaquepaque & 100 \\
\hline La Barca & 100 & Teocaltiche & 100 & Puerto Vallarta & 100 \\
\hline Ameca & 100 & Tonalá & 100 & Zapopan & 100 \\
\hline Tonalá & 100 & Puerto Vallarta & 100 & Ameca & 100 \\
\hline Arandas & 100 & Arandas & 100 & La Barca & 100 \\
\hline El Salto & 100 & Autlán de Navarro & 100 & Zapotlanejo & 100 \\
\hline Tlajomulco de Zúñiga & 100 & Tepatitlán de Morelos & 100 & San Juan de los Lagos & 100 \\
\hline Lagos de Moreno & 100 & Ameca & 100 & Encarnación de Díaz & 100 \\
\hline Zapotlanejo & 100 & Tlajomulco de Zúñiga & 100 & El Salto & 100 \\
\hline Zapotlán el Grande & 100 & Zapopan & 100 & Sayula & 100 \\
\hline Poncitlán & 100 & Zapotlanejo & 100 & Tlajomulco de Zúñiga & 100 \\
\hline Zapopan & 100 & Atotonilco el Alto & 100 & Chapala & 100 \\
\hline Atotonilco el Alto & 100 & Tlaquepaque & 98.55 & $\begin{array}{l}\text { Ixtlahuacán } \\
\text { de los Membrillos }\end{array}$ & 100 \\
\hline Puerto Vallarta & 100 & Lagos de Moreno & 97.55 & Atotonilco el Alto & 100 \\
\hline Autlán de Navarro & 96.91 & La Barca & 92.82 & Zapotlán el Grande & 100 \\
\hline Ayotlán & 95.46 & Ocotlán & 92.44 & Autlán de Navarro & 100 \\
\hline Jocotepec & 95.24 & Tequila & 85.94 & Tomatlán & 100 \\
\hline Tlaquepaque & 89.86 & San Juan de los Lagos & 85.14 & Ayotlán & 100 \\
\hline San Juan de los Lagos & 87.69 & Guadalajara & 72.26 & Ocotlán & 98.01 \\
\hline Tequila & 84.54 & El Salto & 67.54 & Tonalá & 97.73 \\
\hline Ocotlán & 82.99 & Zapotlán el Grande & 64.11 & Lagos de Moreno & 95.47 \\
\hline Tepatitlán de Morelos & 79.28 & & & Poncitlán & 94.98 \\
\hline Chapala & 55.35 & & & Guadalajara & 94.82 \\
\hline Tala & 54.75 & & & Arandas & 94.77 \\
\hline \multirow[t]{7}{*}{ Tamazula de Gordiano } & 52.6 & & & Tepatitlán de Morelos & 92.34 \\
\hline & & & & Tala & 91.18 \\
\hline & & & & Tamazula de Gordiano & 89.82 \\
\hline & & & & Cihuatlán & 84.7 \\
\hline & & & & Tequila & 83.21 \\
\hline & & & & Teocaltiche & 78.05 \\
\hline & & & & Jocotepec & 76.21 \\
\hline Promedio & 90.98 & & 93.16 & & 95.85 \\
\hline Desviacion Estandar & 3.03 & & 2.52 & & 1.22 \\
\hline
\end{tabular}




\section{Anexo B}

Cuadro con el puntaje de eficiencia relativa de los municipios del recorrido intercuartilico

\begin{tabular}{|c|c|c|c|c|c|}
\hline \multicolumn{6}{|c|}{ Recorrido Intercuartilico } \\
\hline \multicolumn{2}{|l|}{1990} & \multicolumn{2}{|l|}{2000} & \multicolumn{2}{|l|}{2010} \\
\hline Unit & Score & Unit & Score & Unit & Score \\
\hline Zapotitlán de Vadillo & 100 & El Grullo & 100 & Villa Hidalgo & 100 \\
\hline San Martín Hidalgo & 100 & Ayotlán & 100 & Zapotlán del Rey & 100 \\
\hline San Sebastián del Oeste & 100 & San Martín Hidalgo & 100 & La Huerta & 100 \\
\hline Talpa de Allende & 100 & Sayula & 100 & Ojuelos de Jalisco & 100 \\
\hline San Juanito de Escobedo & 100 & Tolimán & 100 & $\begin{array}{l}\text { San Ignacio Cerro } \\
\text { Gordo }\end{array}$ & 100 \\
\hline Zapotiltic & 100 & Zapotiltic & 100 & Ayutla & 100 \\
\hline La Huerta & 100 & Unión de San Antonio & 100 & Jamay & 100 \\
\hline Mezquitic & 100 & Ojuelos de Jalisco & 100 & Jalostotitlán & 100 \\
\hline Villa Corona & 100 & Talpa de Allende & 100 & EL Arenal & 100 \\
\hline Mascota & 100 & San Julián & 100 & Degollado & 100 \\
\hline $\begin{array}{l}\text { Cuautitlán de García } \\
\text { Barragán }\end{array}$ & 100 & Degollado & 100 & Magdalena & 100 \\
\hline San Miguel el Alto & 100 & Cabo Corrientes & 100 & San Julián & 100 \\
\hline San Julián & 100 & Zacoalco de Torres & 100 & El Grullo & 100 \\
\hline Villa Hidalgo & 100 & Tototlán & 100 & Teuchitlán & 100 \\
\hline Ojuelos de Jalisco & 97.36 & Ixtlahuacán del Río & 100 & Bolaños & 100 \\
\hline Acatlán de Juárez & 96.94 & Villa Hidalgo & 100 & Zacoalco de Torres & 99.22 \\
\hline Tizapán el Alto & 94.95 & Acatic & 100 & $\begin{array}{l}\text { Ahualulco de Mer- } \\
\text { cado }\end{array}$ & 99.06 \\
\hline Jamay & 94.54 & Mexticacán & 100 & Colotlán & 97.65 \\
\hline Juanacatlán & 93.84 & Jesús María & 100 & San Gabriel & 97.5 \\
\hline Jalostotitlán & 90.77 & Atoyac & 100 & Acatlán de Juárez & 97.18 \\
\hline El Grullo & 87.65 & Tomatlán & 96.35 & Cocula & 96.94 \\
\hline Sayula & 85.69 & $\begin{array}{l}\text { Yahualica de González } \\
\text { Gallo }\end{array}$ & 94.85 & Etzatlán & 96.75 \\
\hline Degollado & 81.36 & Unión de Tula & 90.69 & $\begin{array}{l}\text { Cuautitlán de García } \\
\text { Barragán }\end{array}$ & 96.65 \\
\hline Hostotipaquillo & 80.78 & EL Arenal & 83.43 & San Miguel el Alto & 96.65 \\
\hline Huejuquilla el Alto & 75.09 & Cuquío & 82.78 & San Martín Hidalgo & 96.4 \\
\hline EL Arenal & 72.7 & Pihuamo & 80.27 & Mazamitla & 95.09 \\
\hline San Gabriel & 71.9 & San Gabriel & 80.12 & Tuxpan & 94.62 \\
\hline Teuchitlán & 70.65 & Teuchitlán & 76.82 & Tototlán & 93.68 \\
\hline Tuxpan & 68.24 & Tizapán el Alto & 75.82 & $\begin{array}{l}\text { Unión de San } \\
\text { Antonio }\end{array}$ & 93.41 \\
\hline
\end{tabular}


Economía Informa núm. 393 julio - agosto • 2015

\begin{tabular}{|c|c|c|c|c|c|}
\hline Zacoalco de Torres & 64.96 & Jilotlán de los Dolores & 74.96 & Cuquío & 92.91 \\
\hline Tecolotlán & 62.87 & Tecalitlán & 73.64 & Tapalpa & 92.55 \\
\hline Tomatlán & 62.7 & Tuxpan & 68.83 & Amatitán & 91.65 \\
\hline Cihuatlán & 61.08 & Tonila & 67.03 & Mezquitic & 91.55 \\
\hline Amatitán & 57.98 & Tecolotlán & 64.07 & Cabo Corrientes & 91.28 \\
\hline Teocuitatlán de Corona & 56.25 & Mascota & 63.26 & Unión de Tula & 91.23 \\
\hline $\begin{array}{l}\text { Ixtlahuacán de los Mem- } \\
\text { brillos }\end{array}$ & 56.21 & Ahualulco de Mercado & 62.53 & Villa Corona & 91.02 \\
\hline Pihuamo & 53.54 & Magdalena & 60.66 & Tizapán el Alto & 90.75 \\
\hline Mexticacán & 53.06 & $\begin{array}{l}\text { San Juanito de Esc- } \\
\text { obedo }\end{array}$ & 57.88 & Juanacatlán & 88.66 \\
\hline Tecalitlán & 52.12 & Tenamaxtlán & 55.37 & Casimiro Castillo & 87.8 \\
\hline Villa Purificación & 49.78 & Casimiro Castillo & 53.92 & $\begin{array}{l}\text { San Juanito de Esc- } \\
\text { obedo }\end{array}$ & 87.47 \\
\hline Gómez Farías & 44.85 & & & Tecolotlán & 86.3 \\
\hline Cabo Corrientes & 43.41 & & & Pihuamo & 85.22 \\
\hline Zapotlán del Rey & 42.67 & & & Jesús María & 84.65 \\
\hline Tenamaxtlán & 42.42 & & & Zapotiltic & 83.2 \\
\hline Ahualulco de Mercado & 42.03 & & & $\begin{array}{l}\text { Jilotlán de los Do- } \\
\text { lores }\end{array}$ & 82.69 \\
\hline Jilotlán de los Dolores & 40.34 & & & Acatic & 78.84 \\
\hline Tolimán & 39.16 & & & $\begin{array}{l}\text { Yahualica de } \\
\text { González Gallo }\end{array}$ & 77.89 \\
\hline Atoyac & 36.94 & & & Tolimán & 76.57 \\
\hline Tonila & 36.82 & & & Hostotipaquillo & 76.18 \\
\hline \multirow[t]{13}{*}{ Mazamitla } & 33.72 & & & Zapotitlán de Vadillo & 75.89 \\
\hline & & & & $\begin{array}{l}\text { Teocuitatlán de } \\
\text { Corona }\end{array}$ & 74.92 \\
\hline & & & & Huejuquilla el Alto & 73.59 \\
\hline & & & & Tecalitlán & 73.51 \\
\hline & & & & Atoyac & 73 \\
\hline & & & & Ixtlahuacán del Río & 72.73 \\
\hline & & & & Tenamaxtlán & 72.68 \\
\hline & & & & Mascota & 72.06 \\
\hline & & & & Quitupan & 71.66 \\
\hline & & & & Talpa de Allende & 70.76 \\
\hline & & & & Tonila & 67.98 \\
\hline & & & & Valle de Guadalupe & 64.13 \\
\hline & & & & Villa Purificación & 59.77 \\
\hline Promedio & 73.91 & & 86.58 & & 88.74 \\
\hline Desviacion Estandar & 3.34 & & 2.57 & & 1.44 \\
\hline
\end{tabular}


Anexo C

Cuadro con el puntaje de eficiencia relativa de los municipios del primer cuartil

\begin{tabular}{|c|c|c|c|c|c|}
\hline \multicolumn{6}{|c|}{ Primer Cuartil } \\
\hline \multicolumn{2}{|l|}{1990} & \multicolumn{2}{|l|}{2000} & \multicolumn{2}{|l|}{2010} \\
\hline Unit & Score & Unit & Score & Unit & Score \\
\hline Tonaya & 100 & Valle de Guadalupe & 100 & Tuxcueca & 100 \\
\hline Cuautla & 100 & El Limón & 100 & Amacueca & 100 \\
\hline Ejutla & 100 & Tonaya & 100 & Cuautla & 100 \\
\hline Santa María del Oro & 100 & San Diego de Alejandría & 100 & Atengo & 100 \\
\hline Chiquilistlán & 100 & Valle de Juárez & 100 & Valle de Juárez & 100 \\
\hline Valle de Guadalupe & 100 & Santa María de los Angeles & 100 & Concepción de Buenos Aires & 100 \\
\hline Valle de Juárez & 100 & Guachinango & 100 & La Manzanilla de la Paz & 100 \\
\hline Concepción de Buenos Aires & 100 & Bolaños & 100 & Chimaltitán & 100 \\
\hline Amacueca & 100 & Chimaltitán & 100 & San Diego de Alejandría & 100 \\
\hline Tuxcacuesco & 100 & Ejutla & 100 & Atemajac de Brizuela & 100 \\
\hline Bolaños & 100 & Cuautla & 100 & Guachinango & 100 \\
\hline Huejúcar & 100 & San Sebastián del Oeste & 79.42 & Mixtlán & 100 \\
\hline Cañadas de Obregón & 100 & Amacueca & 72.31 & El Limón & 100 \\
\hline Totatiche & 100 & Chiquilistlán & 71.23 & San Sebastián del Oeste & 99.76 \\
\hline San Diego de Alejandría & 98.98 & Zapotitlán de Vadillo & 67.1 & Atenguillo & 98.87 \\
\hline Atemajac de Brizuela & 96.26 & Tuxcacuesco & 64.46 & Chiquilistlán & 98.85 \\
\hline Atenguillo & 95.33 & Techaluta de Montenegro & 60.82 & San Marcos & 97.87 \\
\hline Atengo & 90.26 & Santa María del Oro & 59.62 & Santa María de los Angeles & 97.72 \\
\hline Tuxcueca & 89.96 & La Manzanilla de la Paz & 59.09 & Juchitlán & 94.5 \\
\hline San Cristóbal de la Barranca & 78.05 & Atenguillo & 54.69 & Huejúcar & 92.49 \\
\hline La Manzanilla de la Paz & 61.49 & Atengo & 53.42 & Tonaya & 89.65 \\
\hline Santa María de los Angeles & 59.08 & & & Techaluta de Montenegro & 84.96 \\
\hline Techaluta de Montenegro & 55.09 & & & Tuxcacuesco & 83.04 \\
\hline San Martín de Bolaños & 51.35 & & & Ejutla & 77.67 \\
\hline \multirow[t]{7}{*}{ San Marcos } & 50.66 & & & Mexticacán & 76.17 \\
\hline & & & & Villa Guerrero & 71.57 \\
\hline & & & & Totatiche & 69.04 \\
\hline & & & & San Cristóbal de la Barranca & 64.98 \\
\hline & & & & Cañadas de Obregón & 64.43 \\
\hline & & & & Santa María del Oro & 59.2 \\
\hline & & & & San Martín de Bolaños & 48.05 \\
\hline Promedio & 89.06 & & 82.96 & & 89.32 \\
\hline Desviacion Estandar & 3.58 & & 4.18 & & 2.72 \\
\hline
\end{tabular}


Economía Informa núm. 393 julio - agosto • 2015

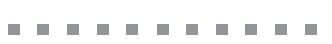

\section{Bibliografía}

Afonso, António y Sónia Fernandes. 2008, Assessing and Explaining the Relative Efficiency of Local Government. The Journal of Socio-Economics 37:1946-1979.

Afonso, António y Sónia Fernandes. 2006, Measuring Local Government Spending Efficiency: Evidence for the Lisbon Region. Regional Studies 40(1):39-53.

Ahmad, Ehtisham, Daniel Hewitt y Edgardo Ruggiero. 1997, Assigning Expenditure Responsibilities. En: Teresa Ter-Minassian (ed.). Fiscal Federalismin Theory and Practice. IMF, Washington, DC.

Ahmad, Ehtisham y Jon Craig.1997, Intergovernmental Transfers. En Teresa TerMinassian (ed.). Fiscal Federalism in Thoery and Practice. International Monetary Fund. Washington, D.C.

Ávila Abud Jorge Alberto y Oscar Javier Cárdenas Rodriguez. 2012, El Impacto de las Transferencias Condicionadas en la Eficiencia Técnica de las Entidades Federativas. En http://www.cefp.gob.mx/portal_archivos/convocatoria/pnfp2012/ pnfp2012_segundolugar.pdf Accesado 13 de septiembre, 2013.

Bonnefoy, Juan Cristóbal y Armijo, Marianela (2005), Indicadores de desempeño en el sector público. Santiago de Chile: CEPAL.

Cabrera, Castellanos Fernando y René Lozano Cortés. 2010, Relaciones Intergubenamentales y el Sistema de Transferencia en México: Una Propuesta de Nivelación Interjurisdicional. Universidad de Quintana Roo.

Charnes, Abraham, William Cooper, y E. Rhodes. 1978, Measuring the Efficiency of Decision-Making Units. European Journal of Operational Research, 2: 429-444.

Charnes, Abraham, William Cooper, y E. Rhodes. 1981, Evaluating Program and Managerial Efficiency: An Application of Data Envelopment Analysis to Program Follow Through. Management Science, 27(6): 668-697.

Diaz, Cayeros Alberto. 2006, Pobreza y Precariedad Urbana en Mexico: Un Enfoque Municipal.cepal:Series Medio Ambiente y Desarrollo, 30:3-57.

Farell, M. J. 1957, The Measurement of Productive Efficency. Journal of the Royal SatatisticalSociety Series, $120(3)$ : 253-278.

Guerrero, Amparán Juan Pablo y Yailen Valdés Palacio. 2000, Manual Sobre la ClasificaciónEconómica del Gasto Público, Programa de Presupuesto y Gasto Público. CIDE, México.

Herrera, Pedro y Pedro Francke. 2007, ¿Por qué algunos Municipios son más Eficientes que Otros? Segunda Parte. Economía y Sociedad, 65:64-71.

Horts, Bettina. 2007, ¿Son Nuestros Municipios Eficientes? Una Aplicación del Análisis Envolvente de Datos. Serie Informe Económico N0. 175, Libertad y Desarrollo. 
Instituto Nacional de Estadística y Geografía (INEGI). 2009, Síntesis Metodológica de la Estadística de Finanzas Públicas Estatales y Municipales, Aguascalientes, Ags.

Mandujano Ramos Nicolás. 2011, Dependencia e Inequidad Tributaria de los Gobiernos Estatales en México. Instituto de Investigaciones Económicas, UNAm, Dimensión Económica, 2(6):20-36.

Murillo-Zamorano, Luis R. 2004, Economic Efficiency and Frontier Techniques. Journal of Economic Surveys, 18(1):33-45.

Noguez, Rivero Gabriel. 2006, Transferencias Intergubernamentales: Su Impacto en el Esfuerzo Recaudatorio y en las Decisiones Presupuestales de los Municipios Mexicanos. Tesis de Maestría. Facultad Latinoamericana de las Ciencias Sociales (Flacso), México, D.F.

Ruiz-Porras, Antonio y Nancy Garcia-Vazquez. 2014, La Planeación de Transferencias hacia los Municipios Jalisciences: Principios de Equidad y no Discriminación. Economía, Sociedad y Territorio, 14(46):601-628.

Peña, Ahumada Jose Antonio. 2012, Manual de Transferencias Federales para Municipios. Instituto Nacional para el Federalismo y el Desarrollo Municipal. Mexico. En http://www.inafed.gob.mx/es/inafed/Manual_de_Transferencias_Federales_ para_Municipios. Accesado 3 de mayo de 2015.

Sempere Jaime y Horacio Sobarzo 1998, Federalismo Fiscal en México. El Colegio de México, México.

Sousa, Maria y Borko Stošić. 2005, Technical Efficiency of the Brazilian Municipalities: Correcting Nonparametric Frontier Measurements for Outliers. Journal of Productivity Analysis, 24(2):157-181.

Št’astná, Lenka y Martin Gregor. 2011, Local Government Efficiency: Evidence from the Czech Municipalities. Institute of Economic Studies, Faculty of Social Sciences Charles University in Prague (working paper).

Trujillo, Salazar Lucía Paola. 2008, Transferencias Intergubernamentales y Gasto Local: Repensando la Descentralización Fiscal Desde una Revisión de la Literatura. Gestión y Política Pública, 7(2):451-486.

Velázquez, Guadarrama Cesar. 2003, Federalismo Fiscal y Transferencias Gubernamentales. En La Descentralización en México: Experiencias y Reflexiones para Orientar la Política Ambiental. Claudia Rodriguez Solórzano (Compiladora). ine-Semarnat, México.

Worthington, Andrew y Brian E. Dollery. 2001, Meassuring Efficiency in Local Government: An Analysis of New South Wales Municipalities'Domestic Waste Management Function. Policy Studies Journal, 29(2):232-249. 